\title{
Integrability, Multi-Solitary Wave Solutions and Riemann Theta Functions Periodic Wave Solutions of the Newell Equation
}

\author{
Chunmei Fang \\ Department of Mathematics and Statistics, Jining Normal University, Ulanqab, China \\ Email: dllgfcmmxl@163.com
}

How to cite this paper: Fang, C.M. (2022) Integrability, Multi-Solitary Wave Solutions and Riemann Theta Functions Periodic Wave Solutions of the Newell Equation. Journal of Applied Mathematics and Physics, 10, 415-424.

https://doi.org/10.4236/jamp.2022.102032

Received: January 23, 2022

Accepted: February 19, 2022

Published: February 22, 2022

Copyright $\odot 2022$ by author(s) and Scientific Research Publishing Inc. This work is licensed under the Creative Commons Attribution International License (CC BY 4.0).

http://creativecommons.org/licenses/by/4.0/

\begin{abstract}
This paper systematically studies the complete integrability of the Newell equation. Using generalized Bell polynomials, the corresponding bilinear equation, bilinear Bäcklund transformation, Lax pair, and multi-shock wave solutions are successfully obtained. In addition, using the multidimensional Riemann theta functions, the periodic wave solutions of the Newell equation are constructed. On this basis, the asymptotic behavior of the periodic wave solution is given, which is the relationship between the periodic wave solution and the solitary wave solution.
\end{abstract}

\section{Keywords}

The Newell Equation, Bäcklund Transformation, Lax Pair, Solitary Wave Solution, Periodic Wave Solution

\section{Introduction}

Since the pioneering introduction of solitons into nonlinear science, many experts and scholars have studied and explored its various aspects. In a great deal of work and efforts of scientists, the main focus has been on the search for exact solutions of nonlinear evolution equations and the study of integrability [1]-[7]. The application of the bilinear method, pioneered by the Japanese mathematician Hirota is very important in nonlinear theory [8]. Nakamura proposed a synthetic approach for constructing multi-periodic wave solutions of nonlinear equations based on the Hirota bilinear method. The advantage of this method is that it only depends on the bilinear form. However, it is not easy to seek a bilinear transformation of a nonlinear evolution equation. It is necessary to make an appropriate transformation, and considerable skill is required to find this 
transformation. To solve this problem, Lambert et al. introduced the concept of the Bell polynomials in algebra to nonlinear differential equations, which made it simple to construct bilinear forms of nonlinear equations [9]. This method not only simplifies the computational complexity but is also practical. Furthermore, it was found that based on the bilinear form, the Bäcklund transformation, Lax pair and infinite conservation laws can be obtained [10] [11]. At present, Bell polynomial theory and the Riemann theta function are extended to superintegrable systems, discrete systems and higher-order integrable systems [12]. In addition, Wang wrote the corresponding package, so that the Bell polynomial theory method became more procedural and simplified [13].

The Newell equation is a very important equation in nonlinear mathematical physics. It is widely used in hydrodynamics, and for coastal wave simulation, nonlinear motion of charged particles in electromagnetic fields, propagation of solitary waves in media, and vibration of a one-dimensional nonlinear lattice. Its general form is

$$
u_{t t}-c_{0}^{2} u_{x x}-\alpha u_{x} u_{x x}-\beta u_{x x x x}=0,
$$

using the homogeneous balance method, the Bäcklund transformation and exact solutions of the Newell equation were presented in [14] [15]. The content of this paper is arranged as follows. In Section 2, the bilinear expression of Newell equation is constructed by using multidimensional Bell polynomials. In Section 3, multiple solitary wave solutions and Riemann theta function periodic wave solutions are obtained by using the bilinear expression of Newell equation. In Section 4, the relationship between periodic wave solution and solitary wave solution is further analyzed, and it is strictly proved that the periodic solution tends to soliton solution under the small amplitude limit. In Section 5, the Bäcklund transformation and Lax pair of Newell equation are derived.

\section{Bilinear Form}

First, we do the following transformation by introducing the potential function $q$

$$
u=m(t) q_{x},
$$

where $m=m(t)$ is a function to be determined, bring the above into Equation (1) you can get

$$
m_{t t} q_{x}+2 m_{t} q_{x, t}+m q_{x, 2 t}-c_{0}^{2} m q_{3 x}-\alpha m_{t}^{2} q_{2 x} q_{3 x}-\beta m q_{5 x}=0,
$$

integrating it with respect to $x$ once, and taking the integral constant to zero, can derive the following equation

$$
m_{t t} q+2 m_{t} q_{t}+m q_{2 t}-c_{0}^{2} m q_{2 x}-0.5 \alpha m_{t}^{2} q_{2 x}^{2}-\beta m q_{4 x}=0,
$$

take $m(t)=\frac{6 \beta}{\alpha}$, then Equation (2) can be written as a combination of $P$-polynomials,

$$
E(q)=P_{2 t}(q)-c_{0}^{2} P_{2 x}-\beta P_{4 x}=0 .
$$


By using the following transformation

$$
q=2(\ln f) \leftrightarrow u=m(t) q_{2 x}=\frac{6 \beta}{\alpha} q_{2 x}=\frac{12 \beta}{\alpha}(\ln f)_{x},
$$

the bilinear form of Equation (1) can be obtained as

$$
\left(D_{t}^{2}-c_{0}^{2} D_{x}^{2}-\beta D_{x}^{4}\right) f \cdot f=0 .
$$

\section{Solitary Wave Solutions and Riemann Theta Functions Periodic Wave Solutions}

In this section, we use the bilinear form of the Newell equation to construct the multi-solitary wave solutions and periodic wave solutions.

\subsection{Multi-Solitary Wave Solutions}

Once the bilinear form of Equation (1) is given, the multi-solitary wave solution of Equation (1) can easily be obtained by using symbolic computation and Hirota method. The multi-solitary wave solutions of the Newell equation can be written as

$$
u=\frac{12 \beta}{\alpha}(\ln f)_{x}
$$

with

$$
\begin{gathered}
f=\sum_{\rho=0,1} \exp \left(\sum_{j=1}^{N} \rho_{j} \eta_{j}+\sum_{1 \leq j<i \leq N} \rho_{i} \rho_{j} A_{i j}\right), \\
\mathrm{e}^{A_{i j}}=-\frac{\left(v_{i}-v_{j}\right)^{2}-c_{0}^{2}\left(\mu_{i}-\mu_{j}\right)^{2}-\beta\left(\mu_{i}-\mu_{j}\right)^{4}}{\left(v_{i}+v_{j}\right)^{2}-c_{0}^{2}\left(\mu_{i}+\mu_{j}\right)^{2}-\beta\left(\mu_{i}+\mu_{j}\right)^{4}}, \\
\eta_{j}=\mu_{j} x+v_{j} t+c_{j}, \quad v_{j}= \pm \sqrt{c_{0}^{2} \mu_{j}^{2}+\beta \mu_{j}^{4}},(1 \leq j<i \leq N),
\end{gathered}
$$

in which $\mu_{j}, v_{j}$ are all free constants, and $\sum_{\rho=0,1}$ sums all possible combinations of $\rho_{i}, \rho_{j}=0,1$.

When $N=1$, one-solitary wave solutions of Equation (1) is given by

$$
u=\frac{12 \beta}{\alpha}\left(1+\mathrm{e}^{\eta}\right)_{x}, \eta=\mu x \pm \sqrt{c_{0}^{2} \mu^{2}+\beta \mu^{4} t}+c,
$$

where $u, c$ are free constants (Figure 1).

\subsection{Riemann Theta Function Periodic Wave Solutions}

Here, we obtain one-periodic wave solution of the Newell equation and analyze its asymptotic properties. To do this, we introduce the following one-Riemann theta function:

$$
\vartheta(\xi, \tau)=\sum_{n=-\infty}^{+\infty} \mathrm{e}^{\pi i n^{2} \tau+2 \pi i n \xi},
$$

in which the phase variable $\xi=k x+\omega t+\varepsilon$, and $\operatorname{Im}(\tau)>0$. 


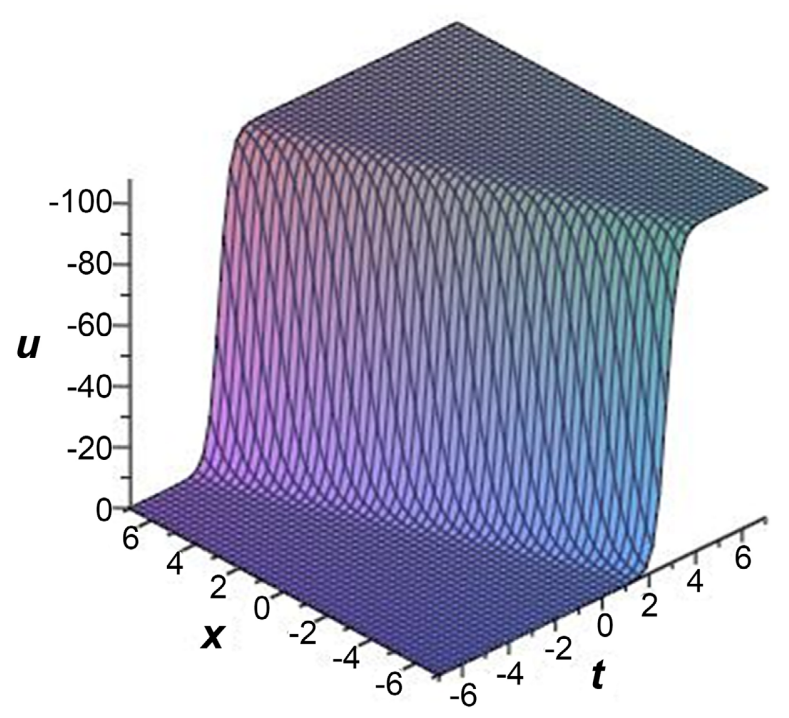

Figure 1. One-solitary wave with parameters: $\alpha=-0.1, \beta=0.5, c_{0}=2, c=1, \mu=1.8$.

To construct the periodic wave solution of Equation (1), we consider the more general form of the bilinear equation as

$$
\left(D_{t}^{2}-c_{0}^{2} D_{x}^{2}-\beta D_{x}^{4}+\delta\right) f \cdot f=0,
$$

where $\delta$ is an arbitrary nonzero constant.

Theorem 1. If $\vartheta(\xi, \tau)$ is a one-Riemann theta function (7) and $\xi=k x+\omega t+\varepsilon$, the Newell equation allows a one-periodic wave solution as follows:

$$
u=\frac{12 \beta}{\alpha} \partial_{x} \ln \vartheta(\xi, \tau)
$$

with

$$
\omega^{2}=\frac{b_{1} a_{22}-b_{2} a_{12}}{a_{11} a_{22}-a_{12} a_{21}}, \delta=\frac{b_{1} a_{21}-b_{2} a_{11}}{a_{12} a_{21}-a_{11} a_{22}},
$$

and

$$
\begin{gathered}
a_{11}=-\sum_{n=-\infty}^{+\infty} 16 n^{2} \pi^{2} \wp^{2 n^{2}}, a_{12}=\sum_{n=-\infty}^{+\infty} \wp^{2 n^{2}}, \\
a_{21}=-\sum_{n=-\infty}^{+\infty} 4(2 n-1)^{2} \pi^{2} \wp^{2 n^{2}-2 n+1}, a_{22}=\sum_{n=-\infty}^{+\infty} \wp^{2 n^{2}-2 n+1}, \\
b_{1}=\sum_{n=-\infty}^{+\infty}\left(256 \beta n^{4} \pi^{4} k^{4}-16 c_{0}^{2} n^{2} \pi^{2} k^{2}\right) \wp^{2 n^{2}}, \\
b_{2}=-\sum_{n=-\infty}^{+\infty}\left(16 \beta(2 n-1)^{4} \pi^{4} k^{4}-4 c_{0}^{2}(2 n-1)^{2} \pi^{2} k^{2}\right) \wp^{2 n^{2}-2 n+1}, \wp=\mathrm{e}^{\pi i \tau},
\end{gathered}
$$

in which the parameters $k, \varepsilon, \tau$ are free.

Proof. For Equation (1), we consider the Riemann theta function solutions of the form (7), according to the Theorem 1 in Ref. [10] and the bilinear equation (8), the parameters $k, \omega, \varepsilon$ satisfy the following system 


$$
\begin{gathered}
\sum_{n=-\infty}^{+\infty}\left(-16 n^{2} \pi^{2} \omega^{2}+16 c_{0}^{2} n^{2} \pi^{2} k^{2}-256 \beta n^{4} \pi^{4} k^{4}+\delta\right) \mathrm{e}^{2 \pi n^{2} \tau}=0, \\
\sum_{n=-\infty}^{+\infty}\left(-4(2 n-1)^{2} \pi^{2} \omega^{2}+4 c_{0}^{2}(2 n-1)^{2} \pi^{2} k^{2}\right. \\
\left.-16 \beta(2 n-1)^{4} \pi^{4} k^{4}+\delta\right) \mathrm{e}^{\pi i\left(2 n^{2}-2 n+1\right) \tau}=0 .
\end{gathered}
$$

According to the representations in (10), the upper expressions are naturally written in the following matrix form:

$$
\left(\begin{array}{ll}
a_{11} & a_{12} \\
a_{21} & a_{22}
\end{array}\right)\left(\begin{array}{c}
\omega^{2} \\
\delta
\end{array}\right)=\left(\begin{array}{l}
b_{1} \\
b_{2}
\end{array}\right)
$$

solving the above linear system results in one-periodic wave solution (9) of Equation (1) (Figure 2).

\section{Asymptotic Analysis}

In this section, we use asymptotic analysis theory to discuss the relationship between one-solitary wave solution and one-periodic wave solution. It is worth noting that if the coefficient matrix, right end vector and solution vector of the system (6.14) can be expanded by a power series with parameter $\wp$, the asymptotic behavior of the periodic wave solution can be analyzed by using the method of small parameter expansion. Via the results from Ref. [10], we expand system (6.14) by parameter $\wp$

$$
\left(\begin{array}{ll}
a_{11} & a_{12} \\
a_{21} & a_{22}
\end{array}\right)=A_{0}+A_{1} \wp+A_{2} \wp^{2}+\cdots
$$

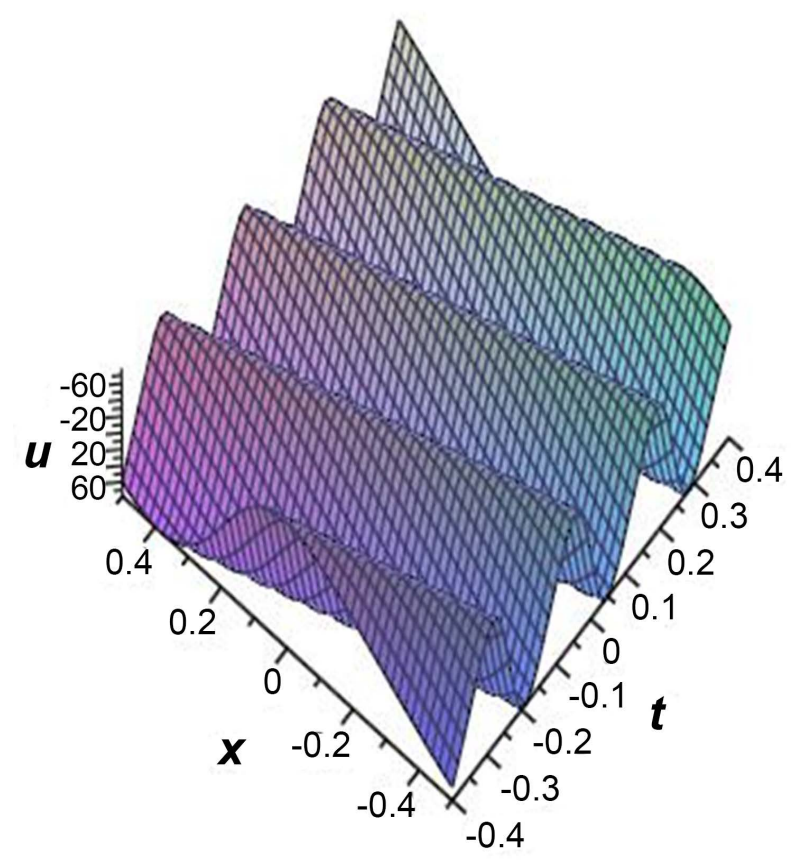

Figure 2. One-periodic wave with parameters: $\alpha=1.1, \beta=2.5, c_{0}=0.1, \tau=0.5 i, k=1, \varepsilon=2$. 


$$
\begin{aligned}
& \left(\begin{array}{l}
b_{1} \\
b_{2}
\end{array}\right)=B_{0}+B_{1} \wp+B_{2} \wp^{2}+\cdots \\
& \left(\begin{array}{c}
\omega^{2} \\
\delta
\end{array}\right)=X_{0}+X_{1} \wp+X_{2} \wp^{2}+\cdots
\end{aligned}
$$

based on Equation (10), rewrite the functions $a_{i j}, b_{j}(i, j=1,2)$ as series expansion with respect to $\wp$

$$
\begin{gathered}
a_{11}=-32 \pi^{2}\left(\wp^{2}+4 \wp^{8}+9 \wp^{18}+\cdots+n^{2} \wp^{2 n^{2}}+\cdots\right), \\
a_{12}=1+2\left(\wp^{2}+\wp^{8}+\wp^{18}+\cdots+\wp^{2 n^{2}}+\cdots\right), \\
a_{21}=-8 \pi^{2}\left(\wp+9 \wp^{5}+25 \wp^{13}+\cdots+(2 n-1)^{2} \wp^{2 n^{2}-2 n+1}+\cdots\right), \\
a_{22}=2\left(\wp+\wp^{5}+\wp^{13}+\cdots+\wp^{2 n^{2}-2 n+1}+\cdots\right), \\
b_{1}=32 \pi^{2}\left(\left(16 \beta \pi^{2} k^{4}-c_{0}^{2} k^{2}\right) \wp^{2}+\left(256 \beta \pi^{2} k^{4}-4 c_{0}^{2} k^{2}\right) \wp^{8}+\cdots\right. \\
\left.+\left(16 \beta n^{4} \pi^{2} k^{4}-c_{0}^{2} n^{2} k^{2}\right) \wp^{2 n^{2}}+\cdots\right), \\
b_{2}=8 \pi^{2}\left(\left(4 \beta \pi^{2} k^{4}-c_{0}^{2} k^{2}\right) \wp+\left(4 \beta(2 n-1)^{4} \pi^{2} k^{4}\right.\right. \\
\left.\left.\quad-c_{0}^{2}(2 n-1)^{2} k^{2}\right) \wp^{2 n^{2}-2 n+1}+\cdots\right),
\end{gathered}
$$

combined with Equations (12) and (13), we get

$$
\begin{gathered}
A_{1}=\left(\begin{array}{cc}
0 & 0 \\
-8 \pi^{2} & 2
\end{array}\right), A_{2}=\left(\begin{array}{cc}
-32 \pi^{2} & 2 \\
0 & 0
\end{array}\right), \\
A_{3}=A_{4}=0, \cdots B_{1}=\left(\begin{array}{c}
0 \\
8 \pi^{2} \Delta_{1}
\end{array}\right), \\
B_{2}=\left(\begin{array}{c}
32 \pi^{2} \Delta_{2} \\
0
\end{array}\right), B_{5}=\left(\begin{array}{c}
0 \\
72 \pi^{2} \Delta_{3}
\end{array}\right), B_{0}=B_{3}=B_{4}=0, \cdots
\end{gathered}
$$

in which $\Delta_{i}$ are presented as follows:

$$
\Delta_{1}=4 \beta \pi^{2} k^{4}-c_{0}^{2} k^{2}, \Delta_{2}=16 \beta \pi^{2} k^{4}-c_{0}^{2} k^{2}, \Delta_{3}=36 \beta \pi^{2} k^{4}-c_{0}^{2} k^{2} .
$$

Then, using Equation (4.15) in Ref. [10], we can calculate the following formula

$$
\begin{gathered}
X_{0}=\left(\begin{array}{c}
-\Delta_{1} \\
0
\end{array}\right), X_{2}=\left(\begin{array}{c}
-8 \Delta_{1} k^{-2} \\
32 \pi k^{-1} \Delta_{1}
\end{array}\right), \\
X_{4}=\left(\begin{array}{c}
-39 k^{-2} \Delta_{1}-9 k^{-1} \Delta_{3} \\
192 \pi^{2} k^{-1} \Delta_{1}
\end{array}\right), X_{1}=X_{3}=0, \cdots
\end{gathered}
$$

combined with Equation (12) and the above expression, we can derive

$$
\begin{gathered}
\omega^{2}=-k^{-1} \Delta_{1}-8 k^{-2} \Delta_{1} \wp^{2}+\left(-64 k^{-3} \Delta_{1}-25 k^{-2} \Delta_{1}-9 k^{-1} \Delta_{3}\right) \wp^{4}+o\left(\wp^{4}\right), \\
\delta=32 \pi k^{-1} \Delta_{1} \wp^{2}+\left(-256 \pi^{2} k^{-2} \Delta_{1}+64 \pi^{2} k^{-1} \Delta_{1}\right) \wp^{4}+o\left(\wp^{4}\right) .
\end{gathered}
$$


Further, rewrite the Riemann theta function $\vartheta(\xi)$ to

$$
\vartheta(\xi)=1+\left(\mathrm{e}^{2 \pi i \xi}+\mathrm{e}^{-2 \pi i \xi}\right) \wp+\left(\mathrm{e}^{4 \pi i \xi}+\mathrm{e}^{-4 \pi i \xi}\right) \wp^{4}+\cdots
$$

with the following transformation

$$
k=\frac{\mu}{2 \pi i}, \varepsilon=\frac{c+\pi \tau}{2 \pi i},
$$

it is easy to get that Equation (17) can be rewritten to

$$
\vartheta(\xi, \tau)=1+\mathrm{e}^{\tilde{\xi}}+\left(\mathrm{e}^{-\tilde{\xi}}+\mathrm{e}^{2 \tilde{\xi}}\right) \wp^{2}+\left(\mathrm{e}^{-2 \tilde{\xi}}+\mathrm{e}^{3 \tilde{\xi}}\right) \wp^{6}+\cdots \rightarrow 1+\mathrm{e}^{\tilde{\xi}},
$$

where $\tilde{\xi}=2 \pi i \xi-\pi \tau=\mu x+2 \pi i \omega t+c$.

Combining Eqs. (17)-(19), we can get

$$
\begin{gathered}
\tilde{\xi} \rightarrow \mu x \pm \sqrt{c_{0}^{2} \mu^{2}+\beta \mu^{4}} t+c=\eta \text {, when } \wp \rightarrow 0, \\
\xi \rightarrow \frac{\eta+\pi \tau}{2 \pi i} \text {, when } \wp \rightarrow 0,
\end{gathered}
$$

which means

$$
\vartheta(\xi, \tau) \rightarrow 1+\mathrm{e}^{\eta} \text {, when } \wp \rightarrow 0 .
$$

According to the above analysis, we can conclude that under the limit condition $\wp \rightarrow 0$, the one-periodic wave solution (9) is exactly inclined to the onesolitary wave solution (6).

\section{The Bäcklund Transformation and Related Lax Pair}

Bilinear Bäcklund transformation plays an important role in constructing solutions of nonlinear equations and characterizing the integrability of given systems. Next, we derive the Bäcklund transformation and related Lax pair of the Newell equation.

Let $q=2 \ln g, q^{\prime}=2 \ln f$ be two different solutions of Equation (1), then we can get

$$
\begin{aligned}
& E\left(q^{\prime}\right)-E(q) \\
& =\left(q^{\prime}-q\right)_{2 t}-c_{0}^{2}\left(q^{\prime}-q\right)_{2 x}-3 \beta\left(q^{\prime}-q\right)_{2 x}\left(q^{\prime}+q\right)_{2 x}-\beta\left(q^{\prime}-q\right)_{4 x} \\
& =0,
\end{aligned}
$$

the above formula can be regarded as the bilinear Bäcklund transformation of Equation (1). Based on this, we introduce two new independent variables

$$
v=\frac{q^{\prime}-q}{2}=\ln (f / g), w=\frac{q^{\prime}+q}{2}=\ln (f g),
$$

according to multidimensional binary Bell polynomials, namely $y$-polynomials [9], the corresponding two-field condition is rewritten as

$$
\begin{aligned}
E\left(q^{\prime}\right)-E(q) & =E(v+w)-E(w-v) \\
& =v_{2 t}-c_{0}^{2} v_{2 x}-6 \beta v_{2 x} w_{2 x}-\beta v_{4 x} \\
& =-\beta \partial_{x} Z_{3 x}(v, w)+R(v, w)=0,
\end{aligned}
$$

where 


$$
R(v, w)=-3 \beta \text { Wronskian }\left[\mathscr{y}_{2 x}(v, w), \mathscr{Z}_{x}(v)\right]+v_{2 t}-c_{0}^{2} v_{2 x} .
$$

In order to represent Equation (20) as a $y$-polynomial Bäcklund transformation, we need to add a constraint condition, we can take this constraint as

$$
k \mathscr{Z}_{2 x}(v, w)+y_{t}(v, w)=\lambda,
$$

in which $k= \pm \sqrt{-3 \beta}$ and $\lambda$ are free parameters.

According to the above constraints, we can get

$$
R(v, w)=\partial_{x}\left[-k \mathscr{Y}_{x, t}(v, w)+\partial_{x}\left(\lambda k-c_{0}^{2}\right) \mathscr{Y}_{x}(v, w)\right],
$$

by the above relations, the two-field condition (20) is decomposed into $y$ -polynomial type Bäcklund transformation as

$$
\begin{aligned}
& k \mathscr{Y}_{2 x}(v, w)+\mathscr{Y}_{t}(v, w)=\lambda, \\
& \partial_{x}\left[-\beta \mathscr{Z}_{3 x}(v, w)-k \mathscr{Y}_{x, t}(v, w)+\left(\lambda k-c_{0}^{2}\right) \mathscr{Y}_{x}(v, w)\right]=0 .
\end{aligned}
$$

On the basis of the relation between $y$-polynomials and Hirota bilinear operator [9], we can directly obtain the bilinear Bäcklund transformation of the Newell equation as

$$
\begin{aligned}
& \left(k D_{x}^{2}+D_{t}-\lambda\right) f \cdot g=0, \\
& \left(-\beta D_{x}^{3}-k D_{x} D_{t}+\left(\lambda k-c_{0}^{2}\right) D_{x}+\delta\right) f \cdot g=0,
\end{aligned}
$$

in which $k= \pm \sqrt{-3 \beta}$ and $\lambda, \delta$ are arbitrary parameters.

Based on related theories of Bell polynomials, and by making use of the Hopf-Cole transformation $v=\ln \psi$, we have

$$
\begin{gathered}
\mathscr{Y}_{x}(v)=\frac{\psi_{x}}{\psi}, \mathscr{Z}_{2 x}(v, w)=q_{2 x}+\frac{\psi_{2 x}}{\psi}, \\
\mathscr{Y}_{x, y}(v, w)=q_{x, y}+\frac{\psi_{x y}}{\psi}, \mathscr{Y}_{y}(v)=\frac{\psi_{y}}{\psi}, \\
\mathscr{Z}_{3 x}(v, w)=3 q_{2 x} \frac{\psi_{x}}{\psi}+\frac{\psi_{3 x}}{\psi}, \mathscr{Y}_{t}(v)=\frac{\psi_{t}}{\psi},
\end{gathered}
$$

thus, the system (21) can be linearized as a Lax pair with parameters $\lambda, \delta$

$$
\begin{aligned}
& \left(k \mathcal{P}_{1}+\partial_{t}\right) \psi=k\left(q_{2 x} \psi+\psi_{2 x}\right)+\psi_{t}=\lambda \psi, \\
& \left(\mathcal{P}_{2}-k \partial_{x t}\right) \psi=\left(-3 \beta q_{2 x}-c_{0}^{2}\right) \psi_{x}-\beta \psi_{3 x}-k q_{x, t} \psi-k \psi_{x, t}+\delta \psi=0 .
\end{aligned}
$$

\section{Conclusion}

In this paper, we use the binary Bell polynomials to study the integrable properties of the Newell equation from many aspects, such as the bilinear equation, bilinear Bäcklund transformation and Lax pair. In Section 3, we use the Riemann theta functions and some of the results in Ref. [10] to obtain the periodic wave solutions of the Newell equation. In addition, by considering the small amplitude limit, we further obtain the asymptotic behaviour between the one-periodic wave solution and the one-solitary wave solution. It is noteworthy that the Hi- 
rota bilinear method, the Riemann theta function and the Bell polynomials play important roles in constructing the solutions of nonlinear evolution equations.

\section{Acknowledgements}

This research was supported by the Higher Educational Scientific Research Projects of Inner Mongolia Autonomous Region (Grant No. NJZY20248; NJZY22307).

\section{Conflicts of Interest}

The author declares that there are no conflicts of interest regarding the publication of this article.

\section{References}

[1] Lü, X. and Ma, W.X. (2016) Study of Lump Dynamics Based on a Dimensionally Reduced Hirota Bilinear Equation. Nonlinear Dynamics, 85, 1217-1222. https://doi.org/10.1007/s11071-016-2755-8

[2] Mao, J.J., Tian, S.F., Zou, L. and Zhang, T.T. (2019) Bilinear Formalism, Lump Solution, Lumpoff and Instant on Rogue Wave Solution of a (3+1)-Dimensional B-Type Kadomtsev-Petviashvili Equation. Nonlinear Dynamics, 95, 3005-3017. https://doi.org/10.1007/s11071-018-04736-2

[3] Ma, W.X. (2021) N-Soliton Solution and the Hirota Condition of a (2+1)-Dimensional Combined Equation. Mathematics and Computers in Simulation, 190, 270-279. https://doi.org/10.1016/j.matcom.2021.05.020

[4] Rao, J.G., Chow, K.W., Mihalache, D. and He, J.S. (2021) Completely Resonant Collision of Lumps and Line Solitons in the Kadomtsev-Petviashvili I Equation. Studies in Applied Mathematics, 147, 1007-1035. https://doi.org/10.1111/sapm.12417

[5] Peng, W.Q., Tian, S.F. and Zhang, T.T. (2018) Analysis on Lump, Lumpoff and Rogue Waves with Predictability to the (2+1)-Dimensional B-Type KadomtsevPetviashvili Equation. Physics Letters A, 382, 2701-2708. https://doi.org/10.1016/j.physleta.2018.08.002

[6] Zhou, Y. (2020) Periodic Solitary Wave Solutions of the (2+1)-Dimensional Variable-Coefficient Caudrey-Dodd-Gibbon-Kotera-Sawada Equation. Open Journal of Applied Sciences, 10, 60-68. https://doi.org/10.4236/ojapps.2020.103005

[7] Dong, Z.Z. and Wang, L. (2018) Solitary Wave Solutions and Rational Solutions for Modified Zakharov-Kuznetsov Equation with Initial Value Problem. Journal of Applied Mathematics and Physics, 6, 949-959. https://doi.org/10.4236/jamp.2018.65081

[8] Hirota, R. (2004) Direct Methods in Soliton Theory. Springer, Berlin. https://doi.org/10.1017/CBO9780511543043

[9] Lambert, F. and Springael, J. (2008) From Soliton Equations to Their Zero Curvature Formulation. Acta Applicandae Mathematicae, 102, 147-178. https://doi.org/10.1007/s10440-008-9209-3

[10] Tian, S.F. and Zhang, H.Q. (2010) Riemann Theta Functions Periodic Wave Solutions and Rational Characteristics for the Nonlinear Equations. Journal of Mathematical Analysis and Applications, 371, 585-608.

https://doi.org/10.1016/j.jmaa.2010.05.070

[11] Ma, P.L., Tian, S.F., Tu, J.M. and Xu, M.J. (2015) On the Integrability and Quasi-Periodic Wave Solutions of the Boussinesq Equation in Shallow Water. European 
Physical Journal Plus, 130, 98. https://doi.org/10.1140/epjp/i2015-15098-0

[12] Dong, C. and Deng, S.F. (2020) The Quasi-Periodic Solutions for the Supersymmetric Variable-Coefficient KdV Equation. Modern Physics Letters B, 34, Article ID: 2050171. https://doi.org/10.1142/S0217984920501717

[13] Miao, Q., Wang, Y.H., Chen, Y. and Yang, Y.Q. (2014) PDEBellII: A Maple Package for Finding Bilinear Forms, Bilinear Bäcklund Transformations, Lax Pairs and Conservation Laws of the KdV-Type Equations. Computer Physics Communications, 185, 357-367. https://doi.org/10.1016/j.cpc.2013.09.005

[14] Yuan, P. and Deng, W.D. (2017) Bäcklund Transformation and Exact Solutions of the Newell Equation. Journal of Jishou University (Natural Science Edition), 38, 1-3.

[15] Shi, Y.Q. (2017) The Exact Solutions of Newell Equation. Journal of Guangxi University of Science and Technology, 28, 6-12. 